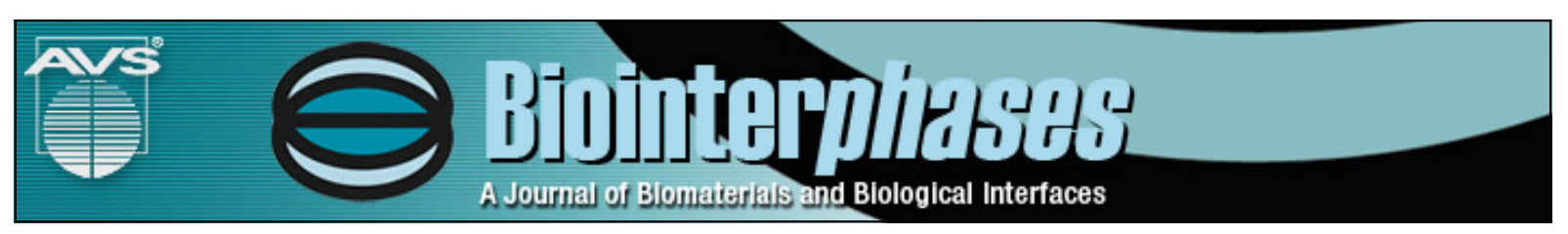

\title{
Hydration force fluctuations in hydrophilic planar systems
}

Matej Kanduč and Roland R. Netz

Citation: Biointerphases 11, 019004 (2016); doi: 10.1116/1.4939101

View online: http://dx.doi.org/10.1116/1.4939101

View Table of Contents: http://scitation.aip.org/content/avs/journal/bip/11/1?ver=pdfcov

Published by the AVS: Science \& Technology of Materials, Interfaces, and Processing

\section{Articles you may be interested in}

Theoretical study on the hydrophobic and hydrophilic hydration on large solutes: The case of phthalocyanines in water

J. Chem. Phys. 143, 044502 (2015); 10.1063/1.4927003

Layering transitions and solvation forces in an asymmetrically confined fluid

J. Chem. Phys. 140, 134704 (2014); 10.1063/1.4869868

Potential of mean force between a large solute and a biomolecular complex: A model analysis on protein flux through chaperonin system

J. Chem. Phys. 135, 185101 (2011); 10.1063/1.3657856

Cooperative hydrophobic/hydrophilic interactions in the hydration of dimethyl ether

J. Chem. Phys. 132, 155102 (2010); 10.1063/1.3367977

Hydration free energies of monovalent ions in transferable intermolecular potential four point fluctuating charge water: An assessment of simulation methodology and force field performance and transferability

J. Chem. Phys. 127, 064509 (2007); 10.1063/1.2771550 


\title{
Hydration force fluctuations in hydrophilic planar systems
}

\author{
Matej Kanduča) \\ Helmholtz-Zentrum Berlin für Materialien und Energie, Hahn-Meitner-Platz 1, D-14109 Berlin, \\ Germany and Fachbereich Physik, Freie Universität Berlin, Arnimallee 14, D-14195 Berlin, Germany \\ Roland R. Netz \\ Fachbereich Physik, Freie Universität Berlin, Arnimallee 14, D-14195 Berlin, Germany
}

(Received 12 November 2015; accepted 16 December 2015; published 8 January 2016)

Utilizing all-atom simulations with explicit solvent, the authors model hydrophilic surfaces interacting across water at a fixed chemical potential. They extract the hydration forces acting between the surfaces and assess force fluctuations as well as interlamellar water number fluctuations. The trends obtained from the simulations are captured by a continuum-based description with effective model parameters. The significance of fluctuations depends on surface hydrophilicity and rigidity. The authors show that the force fluctuations play an important role in kinetic processes in systems with lateral sizes smaller than several tens of nanometers. (C) 2016 American Vacuum Society. [http://dx.doi.org/10.1116/1.4939101]

\section{INTRODUCTION}

Hydrophilic surfaces experience various kinds of forces in the aqueous environment. Besides double layer, van der Waals ( $\mathrm{vdW}$ ), and undulation interactions, which usually dominate at separations above several nanometers, the hydration repulsion becomes a key player at smaller separations. Its short range nature has been experimentally demonstrated in the late $70 \mathrm{~s}$ and 80 s by osmotic stress methods and surface force apparatus measurements. ${ }^{1-7}$ The hydration force controls vital processes in biological matter, e.g., in the structural organization of cells and organelles, as well as in their functionality. It represents an important energy barrier that prevents direct contact and collapse of hydrophilic biological interfaces, and thereby hinders uncontrolled adhesion and fusion of membranes. ${ }^{8}$

The understanding of hydration forces has recently been advanced by computer simulations with explicit water, which are well-suited for tackling such kinds of questions. ${ }^{9-15}$ The hydration force that acts between two surfaces in water highly depends on their polarities and hence on their contact angle. In general, surfaces with lower contact angles give rise to larger hydration repulsion. ${ }^{16}$ On the other hand, the water between hydrophobic surfaces, which are characterized by contact angles above $90^{\circ}$, becomes thermodynamically metastable with respect to cavitation and consequently leads to cavitation-induced long-ranged attraction. ${ }^{9,17-21}$

The behavior of water at hydrophobic surfaces deserved a lot of attention in recent years. ${ }^{2,23}$ It has been realized that water at hydrophobic interfaces is subject to profound density fluctuations, with far-reaching consequences on solvation processes and self-assembly. ${ }^{24-27}$ Furthermore, water exhibits higher compressibility in the vicinity of hydrophobic interfaces, which is gradually lowered and becomes bulklike with increasing hydrophilicity. ${ }^{28-32}$ The local water density fluctuations are sensitive to molecular interactions, and respond to small changes in the surface chemistry,

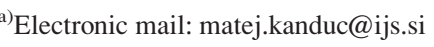

surface conformation, and topology. It has been recently demonstrated that the enhanced water density fluctuations at hydrophobic surfaces can induce bubble nucleation, promoting pathways with significantly lower kinetic free energy barriers of cavitation than predicted by macroscopic theories. ${ }^{33}$ Similar phenomena are present for the kinetics of cavity-ligand binding. ${ }^{34}$

On the other hand, water density fluctuations in hydrophilic systems have been far less investigated. Nevertheless, such density fluctuations can directly influence the hydration repulsion forces, and thereby regulate kinetics by shifting repulsive energy barriers in various biological processes.

In this work, we focus on fluctuations of hydration forces. By utilizing all-atom simulation methods, we model several types of hydrophilic surfaces interacting across water at prescribed chemical potential. We briefly discuss the behavior of interlamellar water and the nature of the resulting hydration interaction. Afterward we focus on the analysis and discussion of the fluctuations of interlamellar water and hydration pressure between the surfaces, their dependence on system size, polarity, and surface stiffness. We corroborate the simulation results by simple continuum-based models, which yield further insight into these fluctuations.

\section{MODEL}

We utilize a simple atomistic model introduced recently, ${ }^{16,35}$ which enables us to study the details of hydration interactions. In this model, we consider two planar polar surfaces that interact across intervening water. The surfaces are composed of anchored ten-carbon-atoms long alkane chains terminated by polar hydroxyl $(-\mathrm{OH})$ head groups facing the water phase in the middle and arranged in a hexagonal lattice with areal density $4.3 / \mathrm{nm}^{2}$. The simulation box has lateral dimensions $5.2 \times 4.5 \mathrm{~nm}$ and is repeated in all three directions via periodic boundary conditions in order to mimic an infinite stack of surfaces. See Fig. 1 for a simulation snapshot. By tuning the model parameters, we can control the adhesive properties of the surfaces. First, by tuning 
the strength of the anchoring potentials on several atoms of the surface molecules, we control the level of head group undulations. By that, we consider two extreme scenarios. With very strong anchors, the surfaces become very stiff and adopt the characteristics of flat crystalline interfaces. On the other hand, weak anchors allow for larger head group fluctuations, resembling self-assembled monolayers. Such soft surfaces exhibit qualitatively similar behavior to membranes. The modeling parameters are given in Table I.

Second, by tuning the polarity of - $\mathrm{OH}$ head groups, we control the hydrophilicity of the surfaces. For that, we scale the partial charges on all head groups by a dimensionless factor $\alpha$. By considering the cases of $\alpha=1$ and $\alpha=0.7$, we model a very hydrophilic and moderately hydrophilic surfaces, respectively. By a combination of two anchoring potentials (i.e., stiff and soft) and two values of the polarity parameter (i.e., $\alpha=1$ and 0.7), we simulate and analyze in total four different types of hydrophilic surfaces.

In order to perform simulations at a prescribed chemical potential of interlamellar water, we use the simulation approach introduced earlier. ${ }^{14,16,35}$ We perform simulations in the canonical NVT ensemble, that is, at constant number of water molecules $(\mathrm{N})$, constant volume $(\mathrm{V})$, and constant temperature $(\mathrm{T})$. At the same time, we measure the normal pressure and the chemical potential of the water phase with a precision of $\pm 0.01 k_{\mathrm{B}} T$. This allows us to numerically evaluate the normal interaction pressure $p$ that corresponds to the

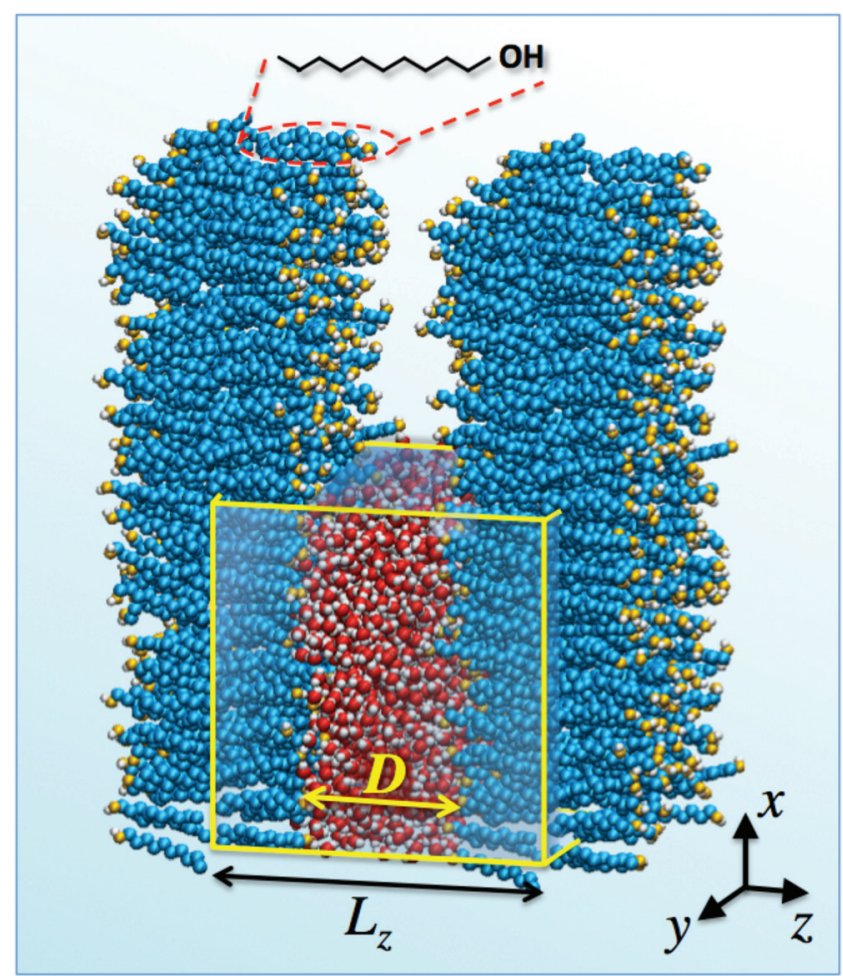

FIG. 1. Snapshot of the simulated system of two soft parallel surfaces consisting of polar molecules interacting across water phase. The simulation box (yellow frame) with dimensions $L_{x}=5.2 \mathrm{~nm}, L_{y}=4.5 \mathrm{~nm}$, and $L_{z}=$ $5.1 \mathrm{~nm}$ is repeated in all three directions via periodic boundary conditions. Water molecules are shown only in the simulation box for clarity.
TABLE I. Summary of the surface model parameters (introduced previously in Ref. 16) and the measured quantities for $\alpha=1$ used in the continuum treatment. The harmonic anchoring potentials with the specified spring constants $k_{x}, k_{y}$, and $k_{z}$ act on the individual atoms listed in the table in each surface molecule. The index at a $\mathrm{C}$ atom labels its successive position counted from the - $\mathrm{OH}$ group. For the detailed determination of the effective surface thickness $b$ and surface compressibility $\chi_{\mathrm{s}}$, see Appendix B. The hydration pressure results for $\alpha=1$ in Fig. 3 are fitted to $p\left(L_{z}\right)=p_{0} \exp \left(-L_{z} / \lambda\right)$ on the entire date range. The fit for the stiff surfaces is not ideal, but needed for the simple model introduced later. The pronounced difference in the pressure amplitudes $p_{0}$ between soft and stiff surfaces appears due to the small surface compressibility of the stiff surfaces.

\begin{tabular}{lcc}
\hline \hline & Soft & Stiff \\
\hline Anchored atoms & $\mathrm{C}_{2}$ & $\mathrm{H}, \mathrm{C}_{1}, \mathrm{C}_{2}, \mathrm{C}_{9}, \mathrm{C}_{10}$ \\
Lateral anchoring potentials $k_{x}, k_{y}$ & $500 \mathrm{~kJ} / \mathrm{mol} / \mathrm{nm}^{2}$ & $500 \mathrm{~kJ} / \mathrm{mol} / \mathrm{nm}^{2}$ \\
Normal anchoring potentials $k_{z}$ & $10 \mathrm{~kJ} / \mathrm{mol}^{2} / \mathrm{nm}^{2}$ & $1000 \mathrm{~kJ} / \mathrm{mol}^{2} \mathrm{~nm}^{2}$ \\
Effective surface thickness, $b$ & $2.9 \mathrm{~nm}$ & $2.9 \mathrm{~nm}$ \\
Surface compressibility, $\chi_{\mathrm{s}}$ & $5.5 \times 10^{-5} \mathrm{bar}^{-1}$ & $0.6 \times 10^{-5} \mathrm{bar}^{-1}$ \\
Decay length, $\lambda$ & $0.22 \mathrm{~nm}$ & $0.11 \mathrm{~nm}$ \\
Amplitude, $p_{0}$ & $5 \times 10^{8} \mathrm{bar}$ & $1.0 \times 10^{15} \mathrm{bar}$ \\
\hline \hline
\end{tabular}

reference chemical potential of bulk water at ambient conditions (i.e., at 1 bar and $300 \mathrm{~K}$ ) with a precision of around \pm 10 bars at large separations. For simulation details, see Appendix A.

\section{RESULTS AND DISCUSSION}

\section{A. Main hydration characteristics of the surfaces}

We first shortly discuss the basic hydration aspects of all four surface types as revealed from the simulations. Quantitative differences among different types of surfaces can be already seen from the density profiles in Fig. 2. Here, the water densities are shown by blue curves (scale on the left) and the head-group oxygen densities by the orange bellshaped curves (in arbitrary units). In the case of soft surfaces, the head group fluctuations are relatively large (wide bell-shaped density profile of the head groups), and consequently, water does not exhibit a particular ordering at the
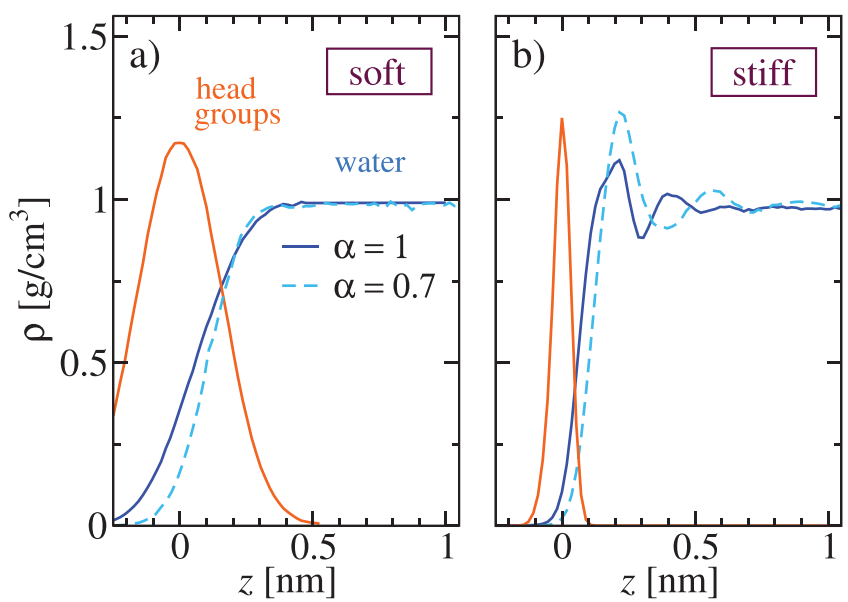

FIG. 2. Density profiles of water (blue) at the highly polar $(\alpha=1)$ and moderately polar $(\alpha=0.7)$ surface in their (a) soft and (b) stiff variants. The headgroup oxygen profiles (orange) are shown in arbitrary units. 
surface. Its density at the interface decays monotonically to zero on a length scale of roughly half a nanometer. On the other hand, the stiffer surfaces cause profound layering of water in their vicinity, ${ }^{16}$ as can be seen from oscillatory density profiles in Fig. 2(b). A second important feature is that water depletes slightly further away from the moderately polar surfaces $(\alpha=0.7)$ as compared with the highly polar surfaces $(\alpha=1)$. Water at less polar surfaces is additionally subject to larger compressibility and larger density fluctuations, as will become important in Sec. III B.

The surface type significantly influences the hydration repulsion between two such surfaces in water, as seen from Fig. 3. In Fig. 3(a), we plot the hydration pressures acting in normal direction between two equal soft surfaces with intervening water at the reference chemical potential as a function of the surface repeat distance $L_{z}$, see Fig. 1 for an illustration of the geometry. The surface-surface separation $D$ can be obtained by subtracting the surface thickness $b$ from the repeat distance, $D=L_{z}-b$, which is for not too high pressures in all cases, $b=2.9 \mathrm{~nm}$ (see Table I). The pressures represented as a function of $D$ have been previously shown in Ref. 16. The pressures reach thousands of bars at close contact and decay monotonically with separation. On the contrary, the stiff surfaces exhibit profound
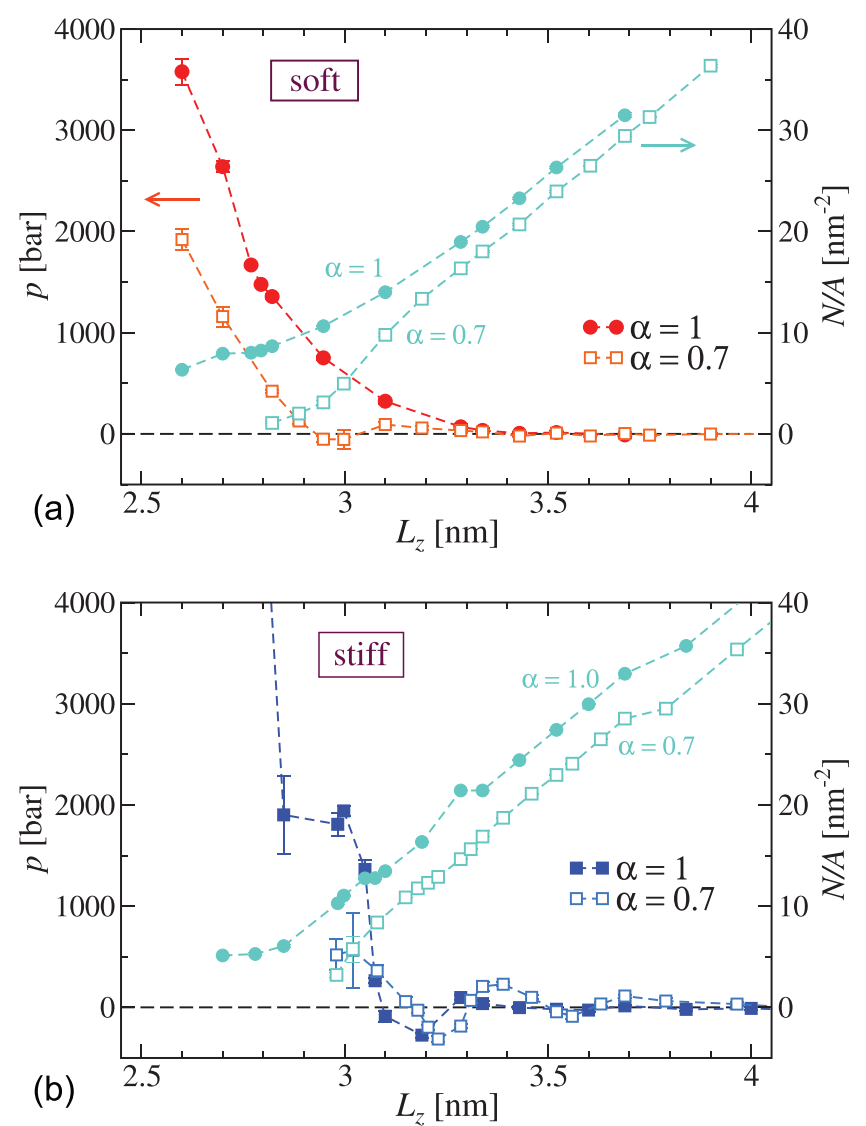

FIG. 3. Hydration pressures as a function of the surface repeat distance $L_{z}$ for two different head-group polarities $\alpha$. The surface thickness in all cases is for not too high pressures $b=2.9 \mathrm{~nm}$. (a) Results for soft surfaces and (b) for stiff surfaces. The corresponding number of interlamellar water molecules is shown by turquoise color data points with the scale on the right. oscillations in the hydration pressures [Fig. 3(b)]. This phenomenon is directly associated to the water layering in Fig. 2(b) and has been discussed in Ref. 16. The amount of interlamellar water between the surfaces is shown by turquoise curves in Fig. 3. It increases with the surface distance and it is slightly higher between more polar surfaces $(\alpha=1)$ as a consequence of different depletion layer thickness, as observed in Fig. 2.

\section{B. Fluctuations evaluated from simulations}

So far, we have considered only the mean values of interaction pressures and interlamellar water amount. In realistic systems, however, the interlamellar water is in equilibrium with an external water reservoir, and therefore, the number of water molecules as well as the interaction pressure are both subject to fluctuations. In the following, we address the question of the nature of these fluctuations and their dependencies on system size and type. We will estimate the intensity of fluctuations in order to assess their importance for finite-size surfaces.

In our canonical simulation approach, the number of water molecules is fixed to $N$ and adjusted to match the reference chemical potential. Consequently, water number fluctuations do not explicitly appear in the simulations. However, by the use of thermodynamic relations, we can numerically predict the fluctuations that would take place in a corresponding grand-canonical scenario. We quantify fluctuations of the interlamellar water number by the variance $\Delta N^{2}=\left\langle N^{2}\right\rangle-\langle N\rangle^{2}$. In general, from a known dependence of the number of particles $N(\mu)$ on the chemical potential, we can predict the number fluctuations as ${ }^{36}$

$$
\Delta N^{2}=k_{\mathrm{B}} T\left(\frac{\partial N}{\partial \mu}\right)_{V, T} .
$$

Here, $N$ is the average number of water molecules at given chemical potential $\mu$. Since in all our systems the temperature $T$ is always constant, we will omit subscripts $T$ from further expressions. By use of the chain rule

$$
\left(\frac{\partial N}{\partial \mu}\right)_{V}=\left(\frac{\partial p}{\partial N}\right)_{V}^{-1}\left(\frac{\partial p}{\partial \mu}\right)_{V}
$$

and using the Maxwell relation

$$
\left(\frac{\partial p}{\partial \mu}\right)_{V}=\left(\frac{\partial N}{\partial V}\right)_{\mu}
$$

we end up with a useful expression for the water number fluctuations

$$
\Delta N^{2}=k_{\mathrm{B}} T\left(\frac{\partial p}{\partial N}\right)_{V}^{-1}\left(\frac{\partial N}{\partial V}\right)_{\mu} .
$$

The two response functions can be easily extracted from our simulations. The required derivative $(\partial p / \partial N)_{V}$ is evaluated from a series of simulations with the same box size but different number of water molecules $N$. The second one 
represents the slope of the interlamellar water content $\left[(\partial N / A) / \partial L_{z}\right]_{\mu}$ given in Fig. 3 .

Figure 4(a) shows the water number fluctuations $\Delta N^{2}$ as predicted by Eq. (4) normalized by the mean value $N$ as a function of surface-surface distance $D$, which is defined as the distance between oxygen density peaks on opposing surfaces. As can be seen, the fluctuations are noticeably larger between the softer surfaces compared with the stiffer ones. Additionally, fluctuations are larger between less polar surfaces, in accordance with general expectations. ${ }^{22,23,31}$

The fluctuations in the number of water molecules between the surfaces induce fluctuations in the hydration pressure. This follows directly from the fact that any change in the number of water molecules by $\mathrm{d} N$ directly influences the hydration pressure between the surfaces via the relation $\mathrm{d} p=(\partial p / \partial N)_{V} \mathrm{~d} N$. This implies $\Delta p^{2}=(\partial p / \partial N)_{V}^{2} \Delta N^{2}$, and by utilizing Eq. (4), we obtain

$$
\Delta p^{2}=k_{\mathrm{B}} T\left(\frac{\partial p}{\partial N}\right)_{V}\left(\frac{\partial N}{\partial V}\right)_{\mu} .
$$
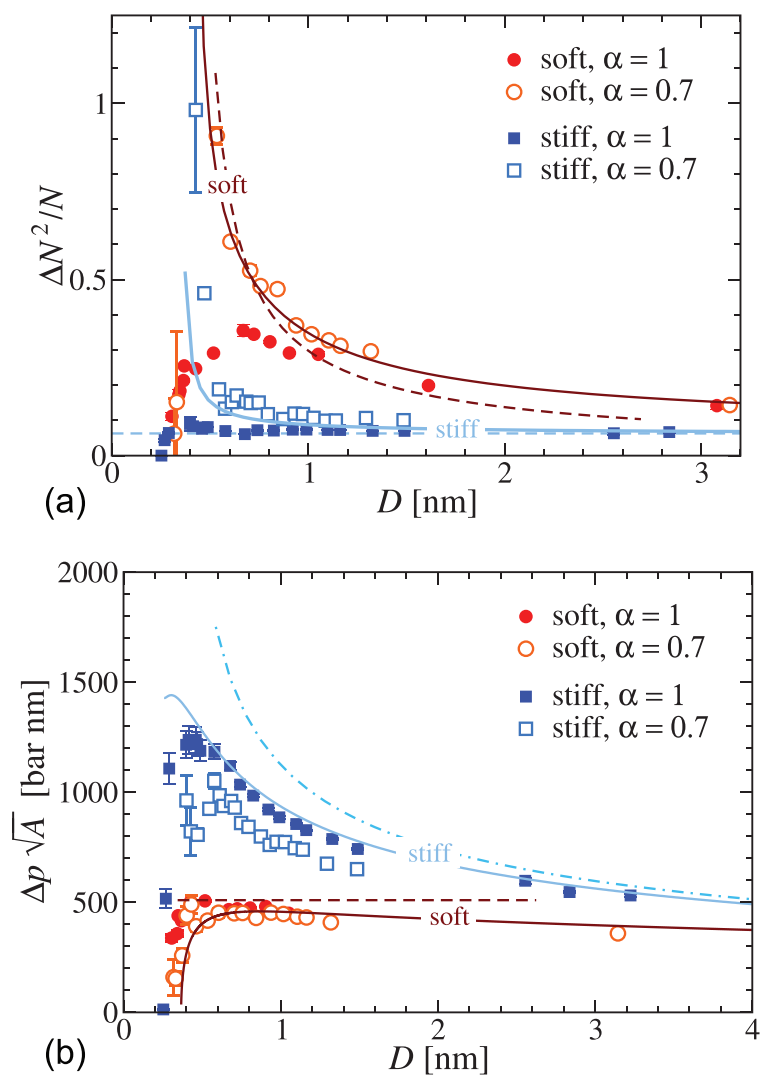

FIG. 4. (a) Fluctuations of the number of interlamellar water molecules. The data points show the values evaluated from simulations via Eq. (4). Solid lines are theoretical predictions for $\alpha=1$ according to Eq. (11) for soft (brown) and stiff (blue) surfaces, respectively. The brown dashed line represents the approximate prediction for the soft case [Eq. (12)], and the blue dashed line represents the approximate prediction for the stiff case [Eq. (13)]. (b) Fluctuations in the hydration pressure. The data points are evaluations from simulations via Eq. (6). Solid lines are theoretical predictions of Eq. (14) $\alpha=1$ for soft (brown) and stiff (blue) surfaces, respectively. The brown dashed line represents the approximate prediction [Eq. (15)] for the soft case. The blue dashed line represents the approximate prediction for the stiff case [Eq. (16)].
Expressing all quantities as intensive ratios (independent of the lateral surface area $A$ ) and taking a square root, we obtain

$$
\Delta p \sqrt{A}=\left[k_{\mathrm{B}} T\left(\frac{\partial p}{\partial N / A}\right)_{L_{z}}\left(\frac{\partial N / A}{\partial L_{z}}\right)_{\mu}\right]^{1 / 2} .
$$

Obviously, the magnitude of the pressure fluctuations $\Delta p$ scales inversely with the lateral size of a system, $\Delta p \propto$ $1 / \sqrt{A}$. That means that smaller planar surfaces are subject to larger pressure fluctuations compared with larger ones. For infinitely large interacting surfaces, the pressure fluctuations vanish all together. Figure 4(b) shows the pressure fluctuations evaluated from all four simulated systems as a function of the surface separation $D$. As is immediately apparent, the pressure fluctuations primarily depend on surface rigidity. Most importantly, stiffer surfaces are subject to larger pressure fluctuations than soft surfaces. The particular data in Fig. 4(b) suggest that the hydration pressure between the stiff polar surfaces $1 \mathrm{~nm}$ apart and with area $A=10 \times 10 \mathrm{~nm}^{2}$ across fluctuates with a magnitude of around 100 bars, and for lateral surface size of $A=100 \times 100 \mathrm{~nm}^{2}$ around 10 bars. In the case of soft surfaces, the fluctuations are reduced to roughly half of that. This gives us a first impression of the importance of pressure fluctuations for a given system size. Thus, for surface sizes below around several tens of nanometers, hydration force fluctuations can become quite sizeable.

Comparing the number and pressure fluctuations in Figs. 4(a) and 4(b), an inverse trend among all four surface types is clearly evident. At first sight, it may seem counterintuitive that the lower the number fluctuations $\Delta N$, the larger the pressure fluctuations $\Delta p$. In fact, the relation between $\Delta N$ and $\Delta p$ can be obtained by eliminating $(\partial p / \partial N)_{V}$ from Eqs. (4) and (6), which yields

$$
\Delta p \Delta N=k_{\mathrm{B}} T\left(\frac{\partial N}{\partial V}\right)_{\mu}
$$

As we will discuss in more detail in Sec. III C, for not too small distances we can approximate $(\partial N / \partial V)_{\mu} \simeq \rho_{\mathrm{w}}$, where $\rho_{\mathrm{w}}$ is the water density, and hence, $\Delta p \Delta N \simeq k_{\mathrm{B}} T \rho_{\mathrm{w}}$. Thus, the number and pressure fluctuations are inversely proportional, which can be intuitively understood as follows. Between soft surfaces the number of particles tends to fluctuate more, since the system can more easily accommodate additional water molecules without a significant increase in pressure. The opposite is true for stiff surfaces, where a few extra molecules increase the pressure significantly and by that prevent further molecules to enter the interlamellar region.

In the next step, we rationalize the observed fluctuation behavior and its distance dependence using a simple continuum description.

\section{Fluctuations predicted by continuum approach}

In order to gain a deeper understanding of the simulation results, we consider a simple continuum description in the 
following. To that end, we link the linear response functions entering Eqs. (4) and (6) to relations based on a simple continuum model.

We first turn our attention to the pressure derivative with respect to the number of water molecules, namely, $(\partial p / \partial N)_{V}$. The pressure change upon inserting additional water molecules at constant volume can be related to the compressibility of the entire system as $\mathrm{d} V / V=-\chi \mathrm{d} p$, where $\mathrm{d} V$ accounts for the volume of the inserted molecules $\mathrm{d} V=\mathrm{d} N / \rho_{\mathrm{w}}$, with $\rho_{\mathrm{w}}$ representing the density of water. The pressure derivative can thus be written as

$$
\left(\frac{\partial p}{\partial N / A}\right)_{L_{z}}=\frac{1}{\rho_{\mathrm{w}} \chi L_{z}}
$$

Here, $\chi$ accounts for the normal isothermal compressibility of the entire system. Our system is composed of a water slab with thickness $D$ and two surfaces with the overall thickness $b$, such that the total repeat distance is $L_{z}=b+D$. From the known isothermal compressibilities of water $\chi_{\mathrm{w}}$ and the surfaces $\chi_{\mathrm{s}}$, the compressibility $\chi$ of the entire system can be approximated as a weighted sum according to $\chi=\left(\chi_{\mathrm{w}} D\right.$ $\left.+\chi_{\mathrm{s}} b\right) / L_{z}$. This gives us an analytical expression for the desired response function, Eq. (8), as

$$
\left(\frac{\partial p}{\partial N / A}\right)_{L_{z}}=\frac{1}{\rho_{\mathrm{w}}\left(\chi_{\mathrm{w}} D+\chi_{\mathrm{s}} b\right)} .
$$

The compressibility of bulk water is $\chi_{\mathrm{w}}=4.5 \times 10^{-5} \mathrm{bar}^{-1}$. We determine the compressibility and effective thickness of the surfaces in independent simulations, where we measure the pressure response of the surfaces to compression. A detailed description is given in Appendix B, with the results listed in Table I. We quantitatively verify the analytic relation Eq. (9) by comparison with simulated results in Appendix C.

The other thermodynamic relation that we describe via a simple continuum model is the change of the number of water molecules with respect to the repeat distance at constant chemical potential, $\left(\partial N / \partial L_{z}\right)_{\mu}$. Performing a few thermodynamic manipulations shown in Appendix D, we obtain

$$
\left(\frac{\partial N / A}{\partial L_{z}}\right)_{\mu}=\rho_{\mathrm{w}}\left[1-\left(\chi_{\mathrm{w}} D+\chi_{\mathrm{s}} b\right) \frac{p(D)}{\lambda}\right] .
$$

Here, the pressure $p(D)$ is related to $p\left(L_{z}\right)$ in Table I. This expression has a simple physical interpretation. At large enough separations $D$, where the hydration pressure $p(D)$ does not significantly contribute to the compression of the surfaces, the change in the repeat distance $\mathrm{d} L_{z}$ results exclusively from the change in the water slab thickness $\mathrm{d} D=\mathrm{d} L_{z}$. The corresponding change in the water molecule number is then $\mathrm{d} N / A=\rho_{\mathrm{w}} \mathrm{d} L_{z}$; hence, $(\partial N / \partial V)_{\mu} \simeq \rho_{\mathrm{w}}$. In contrast, at higher pressures, part of the repeat-distance reduction $\mathrm{d} L_{z}$ goes on the expense of surface compression. Consequently, the interlamellar water amount reduces more slowly with $L_{z}$. This can be seen from the decreasing slopes of the water number at smaller distances in Fig. 3.
Using the derived analytic relations Eqs. (9) and (10), we obtain from Eq. (4) the expression for water number fluctuation as

$$
\frac{\Delta N^{2}}{N}=\frac{\rho_{\mathrm{w}} k_{\mathrm{B}} T}{D}\left(\chi_{\mathrm{w}} D+\chi_{\mathrm{s}} b\right)\left[1-\left(\chi_{\mathrm{w}} D+\chi_{\mathrm{s}} b\right) \frac{p(D)}{\lambda}\right] .
$$

In Fig. 4(a), we plot the prediction of this equation by solid lines for completely polar $(\alpha=1)$ stiff and soft surfaces. Here, we use the measured effective parameters for the compressibilities and hydration pressures listed in Table I. Both lines agree reasonably well with the simulated data, especially for larger distances.

In the case of soft surfaces, where the entire compressibility of the system is governed by the compressibility of the surfaces, $\chi_{\mathrm{w}} D \ll \chi_{\mathrm{s}} b$, and by neglecting the pressure contribution, we obtain

$$
\frac{\Delta N^{2}}{N} \simeq \frac{\rho_{\mathrm{w}} k_{\mathrm{B}} T}{D} \chi_{\mathrm{s}} b .
$$

The brown dashed line in Fig. 4(a) shows the prediction of Eq. (12) for the soft case. The agreement is good at intermediate distances.

On the other hand, in the limit of large enough surface separations or small enough surface compressibility, that is, $\chi_{\mathrm{w}} D \gg \chi_{\mathrm{s}} b$ and when the interaction pressure is not too large, Eq. (11) simplifies to

$$
\frac{\Delta N^{2}}{N} \simeq \rho_{\mathrm{w}} \chi_{\mathrm{w}} k_{\mathrm{B}} T
$$

which is independent of distance $D$. It essentially represents the amount of bulk water fluctuations, $\Delta N^{2} / N \approx 0.063$. This prediction is represented by a horizontal blue dashed line in Fig. 4(a). As can be seen, in the case of stiff surfaces, the water fluctuations are roughly the same as in bulk water even down to very small separations, $D \sim 0.5 \mathrm{~nm}$.

Similarly as for the water number fluctuations, we now derive an expression for the pressure fluctuations using our simple continuum model relations. Plugging the expressions Eqs. (9) and (10) into Eq. (6) yields

$$
\Delta p \sqrt{A}=\sqrt{k_{\mathrm{B}} T}\left[\frac{1}{\chi_{\mathrm{w}} D+\chi_{\mathrm{s}} b}-p(D) / \lambda\right]^{1 / 2} .
$$

It implies that smaller and less compressible surface systems are subject to larger fluctuations in hydration pressure. Large hydration pressures, which are realized at small separations, suppress the fluctuations. The prediction of Eq. (14) is shown by solid lines in Fig. 4(b) for the highly polar $(\alpha=1)$ stiff and soft surfaces.

We now take a closer look at two interesting limiting cases. In the case of soft surfaces, $\chi_{\mathrm{w}} D \ll \chi_{\mathrm{s}} b$, the above expression simplifies to

$$
\Delta p \sqrt{A} \simeq \sqrt{\frac{k_{\mathrm{B}} T}{\chi_{\mathrm{s}} b}},
$$


which does not depend on surface separation. This prediction is shown as a brown dashed line for the soft case in Fig. 4(b) and it agrees well for intermediate separations. It becomes inaccurate for large separations and also clearly fails at close contact. In the case of stiff surfaces or large separation, $\chi_{\mathrm{w}} D \gg \chi_{\mathrm{s}} b$, Eq. (14) reduces to

$$
\Delta p \sqrt{A} \simeq \sqrt{\frac{k_{\mathrm{B}} T}{\chi_{\mathrm{w}} D}} .
$$

This prediction is plotted as a blue dashed line in Fig. 4(b) and turns out to be a good approximation for stiff surfaces at large separations.

We have demonstrated that the qualitative trends of the hydration fluctuations can be well captured by a simple continuum model for the effective compressibility of the composite surface-water slab system. This serves as a useful tool for estimating fluctuations' magnitudes.

Next, we briefly discuss the role of hydration pressure fluctuations for energy barrier crossing. In various situations, the hydration force represents an energy barrier to a closecontact state, such as in the situations of surface adhesion and membrane fusion.

For an explicit illustration of the role of fluctuations in such processes, we consider a simple scenario, where the repulsive hydration force acting between two interfaces is counteracted by an attractive van der Waals-like force at short distances, such that the pressure is given by

$$
p(D)=\tilde{p}_{0} \mathrm{e}^{-D / \lambda}-\frac{C}{D^{3}} .
$$

Here, we employ the parameters in Table I of the soft polar surfaces with $\alpha=1$ for the hydration repulsion part with the amplitude $\tilde{p}_{0}=p_{0} \mathrm{e}^{-b / \lambda}$. For the strength of the attractive part, we choose $C=1 \mathrm{barnm}{ }^{3}$, which is related to the Hamaker constant via $C=H / 6 \pi$ with a typical value for hydrocarbons $H \sim 1 k_{\mathrm{B}} T .^{37}$ The total pressure corresponding to Eq. (17) is plotted in Fig. 5(a) by a blue solid line. The fluctuations in the hydration pressure due to finite surface size are estimated via Eq. (14), and the corresponding pressure range $p \pm \Delta p$ is represented by shaded regions for the surface areas of $A=100,25$, and $10 \mathrm{~nm}^{2}$. We see that significant fluctuations in the pressure around its mean value lead to a temporal decrease in the pressure and therefore to an effective decrease in the free energy barrier.

Bringing such surfaces into the close-contact state requires first to overcome a large energy barrier $w^{*}=\int_{D^{*}}^{\infty} p(D) \mathrm{d} D$, which from Eq. (17) follows as

$$
w^{*}=\lambda \tilde{p}_{0} \mathrm{e}^{-D^{*} / \lambda}-\frac{C}{2 D^{* 2}} .
$$

Here, $D^{*}$ is the distance at which the pressure vanishes, $p\left(D^{*}\right)=0$. In fact, accurate estimates of the adhesion rate would require knowledge of the attempt rate of the barrier crossing events, which is for such complex systems a numerically demanding task. ${ }^{38}$ Therefore, we only predict
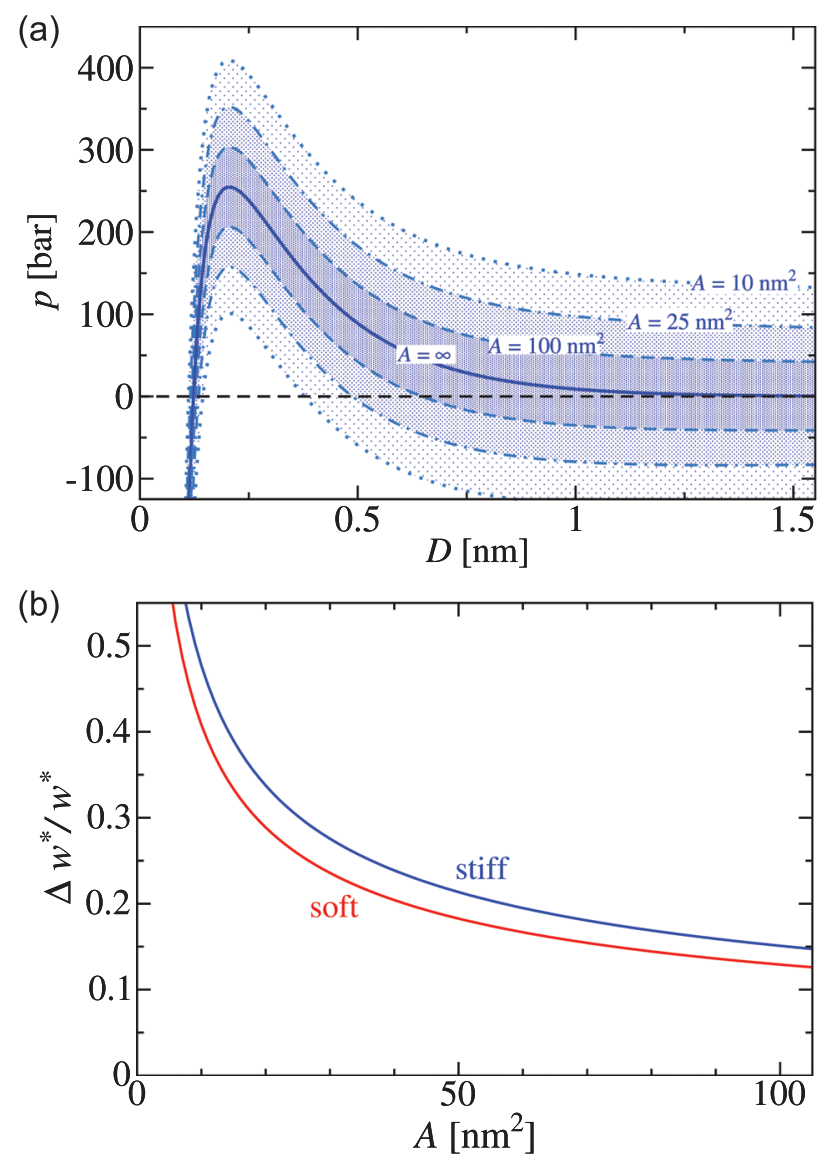

FIG. 5. (a) Hydration pressure of soft polar surfaces counteracted by vdW-like attraction as described by Eq. (17), giving rise to an energy barrier [Eq. (18)]. The shaded stripes represent the estimated range of pressure fluctuations around its mean value for lateral surface areas of $A=100,25$, and $10 \mathrm{~nm}^{2}$, respectively. (b) Estimated relative fluctuations in the free energy barrier $\Delta w^{*} / w^{*}$ from Eqs. (18) and (19) for the soft (red) and stiff (blue) surfaces.

the fluctuations of the free energy barrier height in a heuristic way. The fluctuations can be estimated from the hydration repulsion part in Eq. (18) by replacing the pressure at distance $D^{*}$ by the fluctuation value $\Delta p^{*}$ at $D^{*}$

$$
\Delta w^{*} \approx \lambda \Delta p^{*} .
$$

The ratio $\Delta w^{*} / w^{*}$ that follows from Eqs. (19) and (18) represents relative fluctuations in the free energy barrier height. Assuming $\Delta p^{*} \approx 1 / \sqrt{A} \times 500$ barnm for soft and $\Delta p^{*} \approx$ $1 / \sqrt{A} \times 1300$ barnm for stiff surfaces from Fig. 4(b), we plot the ratio in Fig. 5(b) as a function of surface area $A$. The smaller the interacting areas of the surfaces, the more significant the temporal decrease in the free energy barrier is. Energy fluctuations are significant already for surface areas of $A=100 \mathrm{~nm}^{2}$ and become comparable to the free energy barrier itself for areas around $A=10 \mathrm{~nm}^{2}$. Note that due to the more pronounced pressure fluctuations of stiff surfaces compared with soft surfaces, as seen in Fig. 4(b), the relative energy barrier height decrease is more pronounced for stiff surfaces. We obtain the counter intuitive results that hydration energy barrier height fluctuations are less dramatic for soft surfaces. 


\section{CONCLUSIONS}

Hydrophilic surfaces in water experience large hydration repulsion forces as they approach each other. At the same time, the interacting interfaces are subject to local hydration pressure fluctuations, which can play a significant role in kinetic processes of small systems, such as energy-barrier crossing in adhesion events.

In this article, we analyze the hydration interactions between four different polar surface types. By combining simulation approaches with thermodynamic principles, we are able to extract the basic characteristics of fluctuations of the interlamellar water number and the hydration pressure acting between the surfaces. In general, the stiffer the surfaces, the larger are hydration force fluctuations and smaller are the fluctuations in the number of interlamellar water molecules. The importance of fluctuations dramatically increases with decreasing lateral size of interacting interfaces. Our results suggest that hydration fluctuations become significant for lateral system sizes below several tens of nanometers.

\section{ACKNOWLEDGMENT}

The authors acknowledge funding from the Deutsche Forschungsgemeinschaft via Grant No. SFB 765.

\section{APPENDIX A: SIMULATION DETAILS}

In our simulations, we use the simple point charge/extended (SPC/E) water model ${ }^{39}$ and united-atom parameters taken from the GROMOS forcefield for the surface molecules. ${ }^{40} \mathrm{We}$ reduce the hydrogen-bonding capability among the head groups by slightly increasing their effective sizes. We achieve this by increasing the repulsive part of the Lennard-Jones (LJ) parameter $C_{12}$ between the surface oxygen atoms from the original $1.5 \times 10^{6}$ to $10 \times 10^{6} \mathrm{~nm}^{12} \mathrm{~kJ} / \mathrm{mol}$.

The molecular dynamics simulations are performed with the GROMACS simulation package ${ }^{41}$ in the canonical (NVT) ensemble. The temperature is maintained constant at $T=300 \mathrm{~K}$ by the Berendsen thermostat with a time constant of 1 ps. ${ }^{42}$ Electrostatics is treated using Particle-Mesh-Ewald methods ${ }^{43,44}$ with a $0.9 \mathrm{~nm}$ real-space cutoff. LJ potentials are shifted by a constant offset such that they are zero at their cutoff distance $r_{\mathrm{c}}=0.9 \mathrm{~nm}$. Prior to the production runs, the systems are equilibrated for at least $1 \mathrm{~ns}$. Production runs for measuring the normal pressure and for measuring the LJ part of the excess chemical potential by the Test Particle Insertion method have a duration of $60 \mathrm{~ns}$ for a given separation. Simulations for the Thermodynamic Integration used for measurements of the Coulomb part of the water excess chemical potential have a cumulative duration time of $400 \mathrm{~ns}$ per surface separation.

For a given separation $L_{z}$, the number of water molecules $N$ has to be guessed in the beginning. By measuring the water chemical potential $\mu$ and the normal pressure $p(\mu)$ for the given number of water molecules, we can numerically evaluate the pressure $p\left(\mu_{0}\right)$ that corresponds to the reference chemical potential of bulk water at ambient conditions $\mu_{0}$. If the deviation of the measured chemical potential from the reference value, $\mu-\mu_{0}$, is small enough, the interaction pressure can be evaluated by thermodynamic extrapolation

$$
p\left(\mu_{0}\right)=p+\frac{\mu_{0}-\mu}{v_{\mathrm{w}}} .
$$

Here, $v_{\mathrm{w}}^{-1}=(\partial p / \partial \mu)_{V}=(\partial N / \partial V)_{\mu}$ is related to the change of the system volume upon inserting a water molecule into the system at a constant chemical potential [Eq. (10)]. If the deviation $\mu-\mu_{0}$ is large, one or two additional simulations at iteratively refined values of $N$ are necessary. For a more detailed description of the interaction pressure evaluation, see Ref. 16.

\section{APPENDIX B: COMPRESSIBILITIES OF THE SURFACES}

In order to apply the analytic continuum approach relevant for the atomistic model, we need to extract the effective thickness $b$ of the modeled surfaces as well as their compressibilities $\chi_{\mathrm{s}}$ in the stiff and the soft cases.

We simulate the surfaces without intervening water and monitor the pressure response to an applied compression in $z$-direction. Figure 6(a) shows the perpendicular pressure in the system as a function of the simulation box size $L_{z}$. At
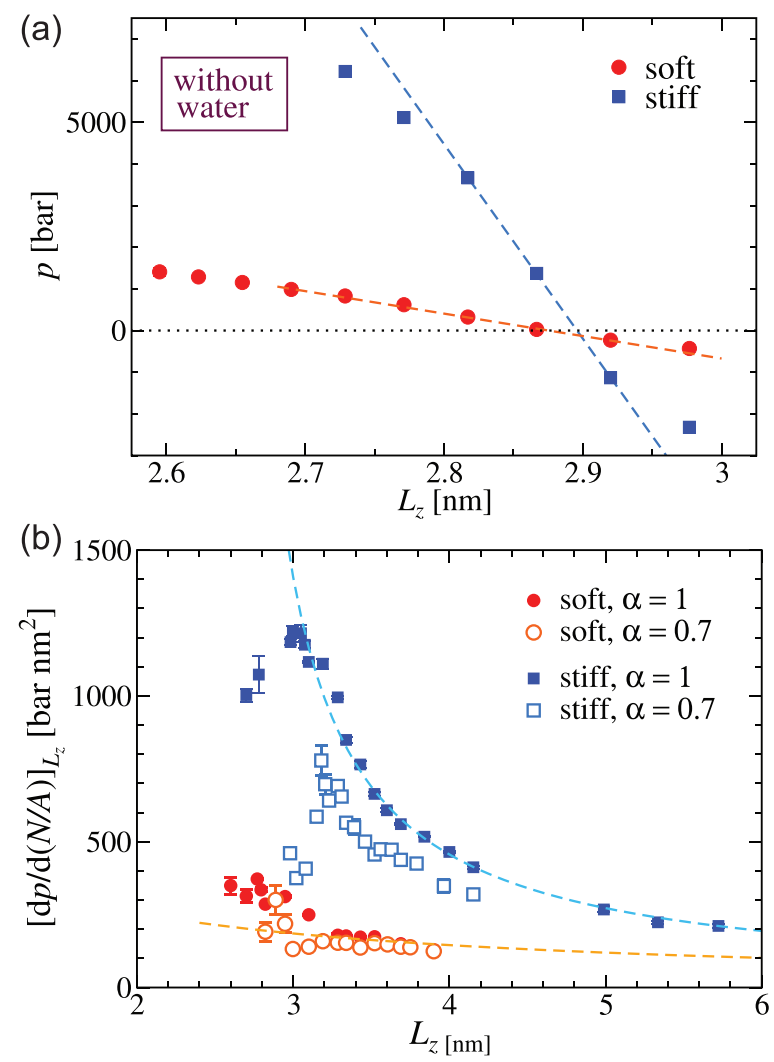

FIG. 6. (a) Perpendicular pressure upon compressing the stiff and the soft polar surfaces with $\alpha=1$ in the absence of interlamellar water. Data points are simulation results. Linear fits around the adhesive state, $p=0$, are shown by dashed lines. (b) Pressure response to a change in the number of water molecules at constant repeat distance. Simulation results for all four surface types are shown by data points. Theoretical predictions of Eq. (9) for $\alpha=1$ are shown by dashed curves for the soft and the stiff cases. 
large distances $L_{z}$, a vacuum gap between the surfaces gives rise to attraction due to dipole-dipole and LJ interactions. At the distance $L_{z} \approx 2.9 \mathrm{~nm}$, the surfaces come into the adhesive state where the attractive forces are balanced by repulsive steric forces, resulting in $p=0$. We use this value to represent the effective thickness of two uncompressed surfaces in the continuum description, $b \approx 2.9 \mathrm{~nm}$. Upon further decrease in the box size, the surfaces undergo compression, resulting in a repulsive pressure $p$ in $z$-direction. For not too large compressions, the pressure scales approximately linearly with $L_{z}$, so that it allows to extract an effective compressibility of the surfaces via the relation $\mathrm{d} V / V=\mathrm{d} L_{z} / L_{z}$ $=-\chi_{\mathrm{s}} \mathrm{d} p$. By linearly fitting the simulation data around $p=0$, we obtain the slopes $\mathrm{d} p / \mathrm{d} L_{z}=4.7 \times 10^{4}$ and $6.2 \times 10^{3} \mathrm{bar} / \mathrm{nm}$, for the stiff and the soft surfaces, respectively. From this, we evaluate the compressibility $\chi_{\mathrm{s}}=$ $\left[b\left(\mathrm{~d} p / \mathrm{d} L_{z}\right)\right]^{-1}$ and obtain $\chi_{\mathrm{s}}=5.5 \times 10^{-5} /$ bar for the soft, and $\chi_{\mathrm{s}}=0.6 \times 10^{-5} / \mathrm{bar}$ for the stiff surface.

\section{APPENDIX C: PRESSURE RESPONSE FUNCTION $[\boldsymbol{\partial} \boldsymbol{p} /(\boldsymbol{\partial} \boldsymbol{N} / \boldsymbol{A})]_{L_{z}}$}

Here, we verify the analytic expression Eq. (9) for the response function by comparing it to simulation data. The response function $[\partial p /(\partial N / A)]_{L_{3}}$ is easily accessible in our simulations. For a given box size $L_{z}$, we perform several NVT simulations with different number of water molecules $N$ around the value that corresponds to the reference chemical potential. At the same time, we measure the corresponding pressure $p(N)$. The desired response function is the derivative of the pressure with respect to $N$ divided by the surface area $A$. The results evaluated from simulations are represented by symbols in Fig. 6(b) as a function of the repeat distance for all four surface types. The dashed lines are the predictions from the analytic continuum model for $\alpha=1$ [Eq. (9)]. The simple model with independently determined $b$ and $\chi_{\mathrm{s}}$ compares reasonably well to the simulation results of fully polar stiff and soft cases at not too small separations. Only below around $L_{z}=3 \mathrm{~nm}$ does the continuum model break down. For the soft surfaces, the total compressibility is governed by the surface compressibility $\chi_{\mathrm{s}}$, and the pressure response function appears to be almost independent of polarity $\alpha$. However, for the stiff surfaces, the total compressibility is dominated by the compressibility of the water slab $\chi_{\mathrm{w}}$, and significant differences appear between the high and the medium polarity.

\section{APPENDIX D: DERIVATION OF THE RELATION $\left[\boldsymbol{\partial}(\boldsymbol{N} / \boldsymbol{A}) / \boldsymbol{\partial} L_{z}\right]_{\mu}$}

Here, we show a detailed derivation of Eq. (10), which represents the change in the interlamellar number of water molecules N/A upon changing the repeat distance $L_{z}$ of the surfaces at prescribed chemical potential $\mu$. Consider the volume of a system as a state function $V(N, p)$. Note that we are disregarding temperature dependence in our notation. The total differential of the volume reads

$$
\mathrm{d} V=\left(\frac{\partial V}{\partial N}\right)_{p} \mathrm{~d} N+\left(\frac{\partial V}{\partial p}\right)_{N} \mathrm{~d} p
$$

Likewise, from the state function $N(\mu, p)$ we derive the total differential of the number of water molecules. By combining the two differentials, we obtain the relation

$$
\left(\frac{\partial V}{\partial N}\right)_{\mu}=\left(\frac{\partial V}{\partial N}\right)_{p}+\left(\frac{\partial V}{\partial p}\right)_{N}\left(\frac{\partial p}{\partial N}\right)_{\mu}
$$

by expanding the second factor of the last term via the chain rule

$$
\left(\frac{\partial p}{\partial N}\right)_{\mu}=\left(\frac{\partial p}{\partial V}\right)_{\mu}\left(\frac{\partial V}{\partial N}\right)_{\mu}
$$

Eq. (D2) is converted into

$$
\left(\frac{\partial V}{\partial N}\right)_{\mu}\left[1-\left(\frac{\partial V}{\partial p}\right)_{N}\left(\frac{\partial p}{\partial V}\right)_{\mu}\right]=\left(\frac{\partial V}{\partial N}\right)_{p} .
$$

We now express the response functions in the above equation by relations from a simple continuum-model. First, we use $(\partial V / \partial p)_{N}=-\chi V$, where $\chi$ is the total compressibility of the system, given by $\chi=\left(\chi_{\mathrm{w}} D+\chi_{\mathrm{s}} b\right) / L_{z}$. Next, the term $\left(\partial p / \partial L_{z}\right)_{\mu}$ represents the rate of change of the hydration pressure with the repeat distance $L_{z}$ at constant chemical potential. Assuming an exponential decaying law for the hydration pressure $p\left(L_{z}\right)=p_{0} \exp \left(-L_{z} / \lambda\right)$, we obtain $\left(\partial p / \partial L_{z}\right)_{\mu}=-p\left(L_{z}\right) / \lambda$. We can express the repeat distance $L_{z}$ in terms of surface-surface distance $D$ as $L_{z}=b+D$. Finally, the term $(\partial V / \partial N)_{p}$ represents the partial volume of a water molecule, namely, the amount by which the system expands upon inserting one water molecule into the system at constant pressure. Neglecting water compressibility, we can express it by the bulk water density $1 / \rho_{\mathrm{w}}$. We furthermore write the volume of the system as $V=A L_{z}$, where $A$ is the surface area and $L_{z}$ the repeat distance of the surfaces. We then finally obtain

$$
\left(\frac{\partial N / A}{\partial L_{z}}\right)_{\mu}=\rho_{\mathrm{w}}\left[1-\left(\chi_{\mathrm{w}} D+\chi_{\mathrm{s}} b\right) \frac{p(D)}{\lambda}\right]
$$

which is Eq. (10) in the main text.

\footnotetext{
${ }^{1}$ V. Parsegian, N. Fuller, and R. Rand, Proc. Natl. Acad. Sci. 76, 2750 (1979).

${ }^{2}$ V. Parsegian and T. Zemb, Curr. Opin. Colloid Interface Sci. 16, 618 (2011).

${ }^{3}$ J. Israelachvili and G. Adams, J. Chem. Soc., Faraday Trans. 1 74, 975 (1978).

${ }^{4}$ J. N. Israelachvili and R. M. Pashley, Nature 306, 249 (1983).

${ }^{5}$ J. N. Israelachvili and H. Wennerström, Langmuir 6, 873 (1990).

${ }^{6}$ J. Israelachvili and H. Wennerström, Nature 379, 219 (1996).

${ }^{7}$ J.-P. Chapel, Langmuir 10, 4237 (1994).

${ }^{8}$ R. Lipowsky, Nature 349, 475 (1991).

${ }^{9}$ D. Bratko, R. A. Curtis, H. W. Blanch, and J. M. Prausnitz, J. Chem. Phys. 115, 3873 (2001).

${ }^{10}$ U. Essmann, L. Perera, and M. L. Berkowitz, Langmuir 11, 4519 (1995).

${ }^{11}$ C. Eun and M. L. Berkowitz, J. Phys. Chem. B 113, 13222 (2009).
} 
${ }^{12}$ A. Pertsin, D. Platonov, and M. Grunze, Langmuir 23, 1388 (2007).

${ }^{13}$ A. Pertsin and M. Grunze, J. Chem. Phys. 137, 054701 (2012).

${ }^{14}$ E. Schneck, F. Sedlmeier, and R. R. Netz, Proc. Natl. Acad. Sci. 109, 14405 (2012).

${ }^{15}$ M. Kanduč and R. R. Netz, Proc. Natl. Acad. Sci. 112, 12338 (2015).

${ }^{16}$ M. Kanduč, E. Schneck, and R. R. Netz, Chem. Phys. Lett. 610, 375 (2014).

${ }^{17}$ J. Wood and R. Sharma, Langmuir 11, 4797 (1995).

${ }^{18}$ H. K. Christenson and P. M. Claesson, Science 239, 390 (1988).

${ }^{19}$ A. Wallqvist and B. J. Berne, J. Phys. Chem. 99, 2893 (1995).

${ }^{20}$ K. Lum, D. Chandler, and J. D. Weeks, J. Phys. Chem. B 103, 4570 (1999).

${ }^{21}$ K. Leung, A. Luzar, and D. Bratko, Phys. Rev. Lett. 90, 065502 (2003).

${ }^{22}$ B. J. Berne, J. D. Weeks, and R. Zhou, Annu. Rev. Phys. Chem. 60, 85 (2009).

${ }^{23}$ S. N. Jamadagni, R. Godawat, and S. Garde, Annu. Rev. Chem. Biomol. Eng. 2147 (2011).

${ }^{24}$ R. Godawat, S. N. Jamadagni, and S. Garde, Proc. Natl. Acad. Sci. 106, 15119 (2009).

${ }^{25}$ A. J. Patel, P. Varilly, and D. Chandler, J. Phys. Chem. B 114, 1632 (2010).

${ }^{26}$ A. J. Patel, P. Varilly, S. N. Jamadagni, H. Acharya, S. Garde, and D. Chandler, Proc. Natl. Acad. Sci. 108, 17678 (2011).

${ }^{27}$ A. J. Patel, P. Varilly, S. N. Jamadagni, M. F. Hagan, D. Chandler, and S. Garde, J. Phys. Chem. B 116, 2498 (2012).

${ }^{28}$ S. I. Mamatkulov, P. K. Khabibullaev, and R. R. Netz, Langmuir 20, 4756 (2004).

${ }^{29}$ H. Acharya, S. Vembanur, S. N. Jamadagni, and S. Garde, Faraday Discuss. 146, 353 (2010).
${ }^{30}$ S. Sarupria and S. Garde, Phys. Rev. Lett. 103, 037803 (2009).

${ }^{31}$ N. Giovambattista, P. J. Rossky, and P. G. Debenedetti, Phys. Rev. E 73, 041604 (2006).

${ }^{32}$ G. Hummer, S. Garde, A. E. Garcia, M. E. Paulaitis, and L. R. Pratt, J. Phys. Chem. B 102, 10469 (1998).

${ }^{33}$ R. C. Remsing, E. Xi, S. Vembanur, S. Sharma, P. G. Debenedetti, S. Garde, and A. J. Patel, Proc. Natl. Acad. Sci. 112, 8181 (2015).

${ }^{34}$ P. Setny, R. Baron, P. Michael Kekenes-Huskey, J. A. McCammon, and J. Dzubiella, Proc. Natl. Acad. Sci. 110, 1197 (2013).

${ }^{35}$ M. Kanduč, A. Schlaich, E. Schneck, and R. R. Netz, Adv. Colloid Interface Sci. 208, 142 (2014).

${ }^{36}$ D. Chandler, Introduction to Modern Statistical Mechanics (Oxford University, New York, 1987).

${ }^{37}$ V. A. Parsegian, Van der Waals Forces: A Handbook for Biologists, Chemists, Engineers, and Physicists (Cambridge University, Cambridge, 2005).

${ }^{38}$ B. Kowalik, T. Schubert, H. Wada, M. Tanaka, R. R. Netz, and E. Schneck, J. Phys. Chem. B 119, 14157 (2015).

${ }^{39}$ H. J. C. Berendsen, J. R. Grigera, and T. P. Straatsma, J. Phys. Chem. 91, 6269 (1987).

${ }^{40}$ C. Oostenbrink, A. Villa, A. E. Mark, and W. F. Van Gunsteren, J. Comput. Chem. 25, 1656 (2004).

${ }^{41}$ D. Van Der Spoel, E. Lindahl, B. Hess, G. Groenhof, A. E. Mark, and H. J. C. Berendsen, J. Comput. Chem. 26, 1701 (2005).

${ }^{42}$ H. J. C. Berendsen, J. P. M. Postma, W. F. van Gunsteren, A. DiNola, and J. R. Haak, J. Chem. Phys. 81, 3684 (1984).

${ }^{43}$ T. Darden, D. York, and L. Pedersen, J. Chem. Phys. 98, 10089 (1993).

${ }^{44}$ U. Essmann, L. Perera, M. L. Berkowitz, T. Darden, H. Lee, and L. G. Pedersen, J. Chem. Phys. 103, 8577 (1995). 